Research Article

\title{
Geometry Optimization Calculations for the Elasticity of Gold at High Pressure
}

\author{
E. Güler and M. Güler \\ Hitit University, Department of Physics, 19030 Corum, Turkey \\ Correspondence should be addressed to E. Güler; eguler71@gmail.com
}

Received 2 May 2013; Revised 18 September 2013; Accepted 23 September 2013

Academic Editor: Augusto Deus

Copyright ( 2013 E. Güler and M. Güler. This is an open access article distributed under the Creative Commons Attribution License, which permits unrestricted use, distribution, and reproduction in any medium, provided the original work is properly cited.

We present embedded atom method based geometry optimization aspects of pressure effect on some elastic and mechanical properties of gold. During study, we determined the pressure dependency of equilibrium volume, typical cubic elastic constants, bulk modulus, shear modulus, young modulus, and elastic wave velocities of the considered metal with varying pressure between $0 \mathrm{GPa}$ and $1000 \mathrm{GPa}$. Finally, we carried out a benchmark between our results and other available theoretical calculations and experimental data. The results of the study mutually agree with the previous findings and provide a deeper outlook for high pressure behavior of the studied metal.

\section{Introduction}

The effect of pressure on the propagation of elastic waves in materials is essential for predicting and understanding some physical properties comprising the interatomic forces, mechanical stability, phase transition mechanisms, dynamic fracture, earthquakes, and the internal structures of Earth. Unfortunately, there is not much experimental information about the elasticity of solids at high pressure, since measurement of the elastic constants is challenging under high pressure.

The two traditional experimental methods for moderate pressures are ultrasonic technique and Brillouin spectroscopy measurements. From these methods, ultrasonic methods utilize a few gigapascals (GPa) where Brillouin spectroscopy measurements are limited to $25 \mathrm{GPa}$ [1]. Furthermore, gold $(\mathrm{Au})$ is often used as an internal pressure calibrant for high pressure studies, because of its stable nature over wide temperature and pressure ranges. $\mathrm{Au}$ is also chemically inert, compressible, and displays a simple X-ray diffraction pattern. However, as reported by Matsui there has been much debate especially on the pressure-volume-temperature $(P-V$ $T$ ) equation of state (EOS) of $\mathrm{Au}$, even at room temperature. Also, estimated pressure differences between proposed EOSs often reach more than $10 \%$ with respect to experiments
[2]. Therefore, to overcome the experimental limitations, computational researches on the high pressure characteristics of $\mathrm{Au}$ are particularly critical for ongoing academic studies and still current research topics [3-6].

Over the past few decades, computer simulation techniques have become an important tool in science as an aid to the interpretation of experimental data for yielding an atomic level model [7]. Notably, two popular calculation methods, namely, classical physics based molecular dynamics (MD) and quantum-mechanics based density functional theory (DFT) methods, play a major role on the materials science and condensed matter scene to get reliable results on the physical properties of metals being dependent to accurate interatomic potentials [8]. For instance, embedded atom method (EAM) and its potentials are well-known schemes for the atomistic simulations of metallic materials [9-11]. In the early 1980s, Daw and Baskes introduced EAM for calculating the ground state properties of realistic metal systems to remove the insufficiency of pairwise potentials [12], and, afterwards, researchers applied this semiemprical method extensively with various types of many improved body potentials for distinct physical problems in metallic systems. Exemplarily, Finnis-Sinclair type EAM potentials adequately explain the elastic and defect properties of base centered cubic (bcc) metals $[13,14]$, whereas Sutton-Chen 


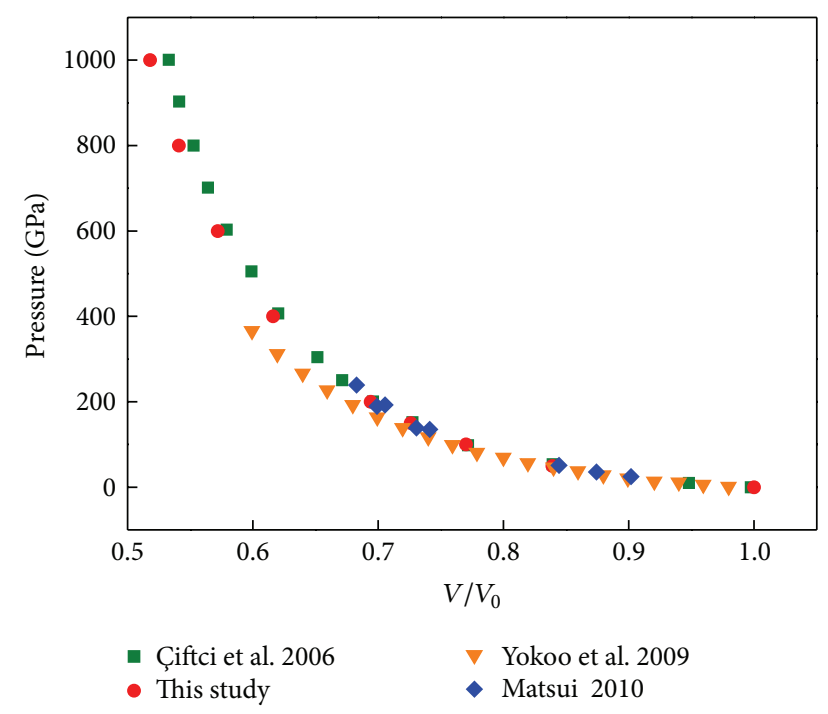

Figure 1: A comparison for the $P-V$ equation of state for Au between $0 \mathrm{GPa}$ and $1000 \mathrm{GPa}$.

type EAM is a proper alternative for face centered cubic (fcc) metals for atomistic calculations [15]. Noteworthy to mention here, the use of EAM with geometry optimization calculations with General Utility Lattice Program (GULP) code reveals veracious research results on some versatile features of metals such as bulk modulus, elastic constants, and elastic moduli $[16,17]$.

In the present study, we focus on clarifying the individual high pressure elastic constants of Au by EAM based geometry optimization calculations. The main purpose of this study is not only to provide results that can be used to evaluate quantitatively the high pressure elasticity of $\mathrm{Au}$ from $0 \mathrm{GPa}$ up to $1000 \mathrm{GPa}$, but also to contribute to enlightening the abovementioned doubts on Au.

\section{Computational Procedure}

During this computational study, we performed all calculations using General Utility Lattice Program (GULP) code 4.0. This simulation code allows performing wide-range property calculations for 3D periodic solids, 2D surfaces and gas phase clusters, and so forth. Besides, this code also allows the use of two-body, three-body, four-body, six-body, and many body (EAM) potentials depending on demands of research. Most calculations in GULP code consist of the optimization of a trial structure to the local energy minimum, under given conditions of pressure and temperature. Moreover, several types of standard minimization techniques are available in GULP. The two common techniques are to optimize the related structures at constant pressure, in which all internal and cell variables are included or at constant volume, where the unit cell remains frozen $[16,17]$. So, we applied a constant pressure optimization for the Au. The geometry of the studied cells was optimized by the Newton-Raphson method [21] based on the Hessian matrix calculated from the second derivatives. The Hessian matrix is recursively updated during optimization using the Broyden-Fletcher-Goldfarb-Shanno algorithm [22-25]. We employed Sutton-Chen type EAM potential to study fcc Au metal, under periodic boundary conditions with a temperature configuration at $T=0$ Kelvin. Then, we increased the pressure from $0 \mathrm{GPa}$ to $1000 \mathrm{GPa}$ in the steps of $200 \mathrm{GPa}$ with a lattice constant $a_{0}=4.08 \AA$ for Au. Following the EAM based optimization results for $\mathrm{Au}$, we particularly concentrated on some elastic parameters such as typical cubic elastic constants $\left(C_{11}, C_{12}\right.$, and $\left.C_{44}\right)$, bulk modulus $(B)$, shear modulus $(G)$, young modulus $(E)$, longitudinal sound velocity $\left(V_{L}\right)$, and shear velocity $\left(V_{T}\right)$ of this metal for the studied pressure range where all these physical parameters are circumstantiated in very recent study of authors [26]. At the end of our calculations we presented a benchmark between our results and earlier experimental and DFT findings of fcc Au.

\section{Results and Discussion}

Since it is not possible get data on the EOS of $\mathrm{Au}$ up to $1000 \mathrm{GPa}$ experimentally, we deliberately focused on the former theoretical results from the literature. Figure 1 shows $P-V$ diagram of Au. In Figure 1 the circle symbols represent our present calculation data where squares show the molecular dynamics results of Çiftci et al. [5]. In addition, diamonds symbols stand for Matsuis data [2], and triangles belong to Yokoo et al. calculations [27]. As it is obvious from Figure 1, our present results on the $P-V$ diagram of $\mathrm{Au}$ are very close to molecular dynamics data in [5] and cover both the Matsuis findings and Yokoo's data in which Yokoo and his coworkers calculations also subsumed the electronic-free energy contribution. As a result, our data strongly supports previous findings on the EOS of $\mathrm{Au}$ and show significant similarities on the $P-V$ curve.

Figure 2 represents the typical elastic constants $\left(C_{11}\right.$, $C_{12}$, and $C_{44}$ ) of $\mathrm{Au}$ at the pressure interval between $0 \mathrm{GPa}$ and $150 \mathrm{GPa}$ where the authors could be able to make a comparison between current results and former experimental data and DFT values. The closed circles in Figure 2 indicate the present calculations while open-crossed circles denote experimental data of Duffy et al. [28] and squares show Greeff and Graf DFT data [29]. It is quite clear in Figure 2 that our elastic constant data, appears in the range of both experimental and DFT data except the little disagreement of $C_{44}$. Additionally, Figure 3 outlines the pressure dependency of the elastic constants in the $0 \mathrm{GPa}-1000 \mathrm{GPa}$ range obtained during this research. As seen in Figure 3, the calculated values of $C_{11}, C_{12}$, and $C_{44}$ elastic constants are positive and exhibit a smooth increment as a function of the increasing pressure. Besides, the increment of the elastic constant $C_{11}$ is higher than both elastic constants $C_{12}$ and $C_{44}$. Physically, $C_{11}$ describes the longitudinal elastic behavior, whereas $C_{12}$ and $C_{44}$ portray the off-diagonal and shear elastic characteristic of cubic crystals related with shearing, respectively. So, a longitudinal strain produces a change in volume without a change in shape. This volume change is highly related to pressure and thus reflects a large change in $C_{11}$. On the other hand, a transverse strain or shearing causes a change in shape 


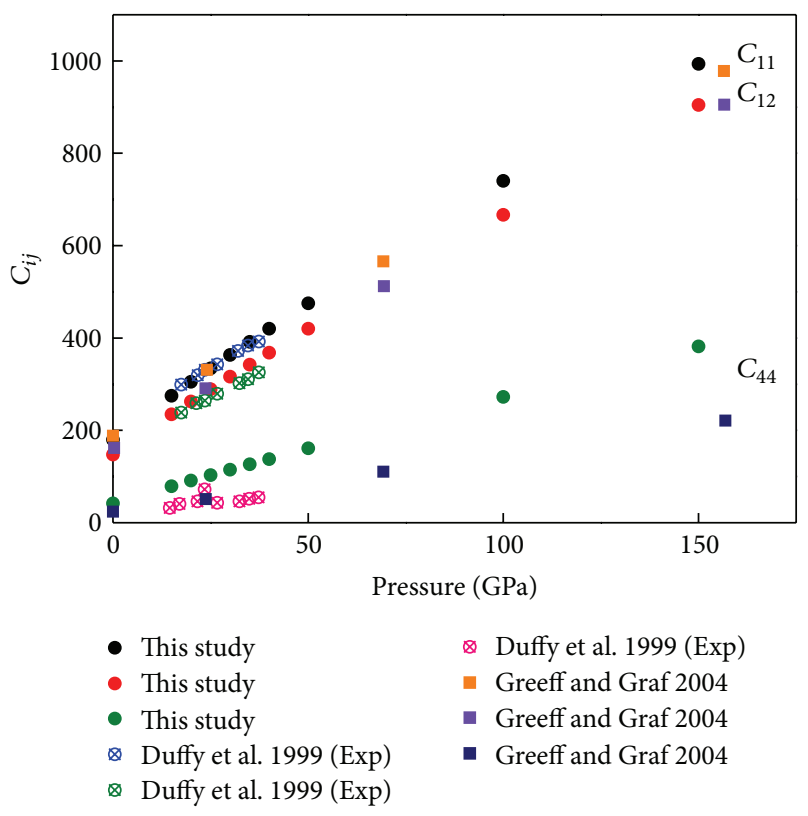

FIgURE 2: Comparing typical cubic elastic constants of $\mathrm{Au}$ as a function of pressure.

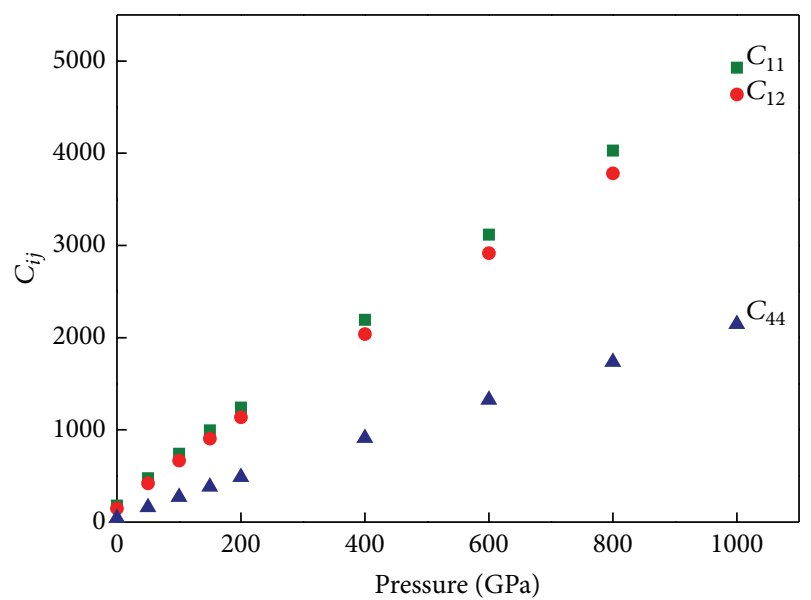

Figure 3: Typical cubic elastic constants of $\mathrm{Au}$ from $0 \mathrm{GPa}$ to $1000 \mathrm{GPa}$.

without a change in volume. Therefore, $C_{12}$ and $C_{44}$ are less sensitive to pressure when compared with $C_{11}$.

Figure 4 displays the behavior of three elastic moduli $(B$, $G$, and $E$ ) of $A$ u for the applied pressure range. From the prevalent physical definition of bulk modulus $B=\Delta P / \Delta V$, an increment for $B$ is expected due to its direct proportionality to applied pressure. Thus, it is visible in Figure 4 that bulk modulus of Au represents a straight increment as expected. In this regard, the other elastic moduli $G$ and $E$ depending strictly on bulk modulus also expose similar behavior in Figure 4 . The behavior of these curves is also consistent within the former results of moderating pressures in metals and alloys.

Pressure effect on the longitudinal sound velocity $\left(V_{L}\right)$ and shear velocity $\left(V_{T}\right)$ of Au up to $1000 \mathrm{GPa}$ can be seen in

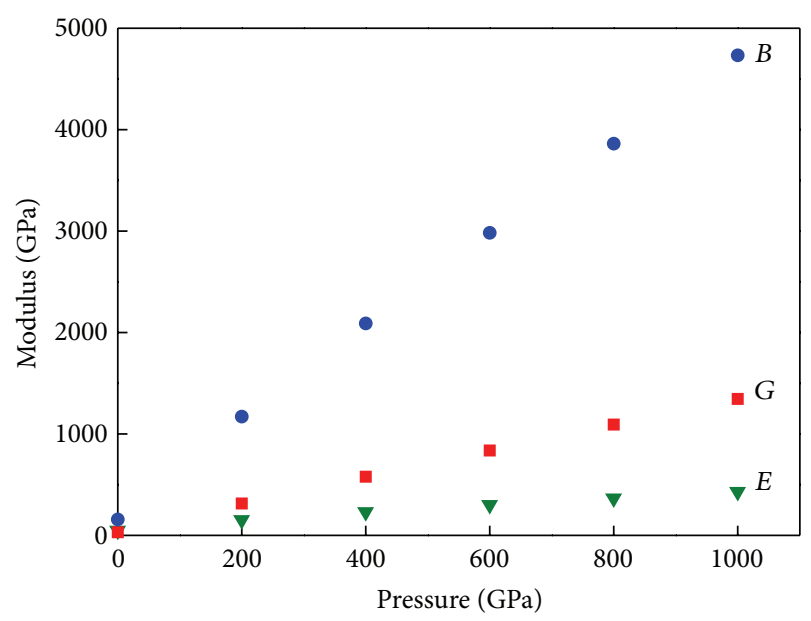

FIgURE 4: Bulk modulus, shear modulus, and young modulus of $\mathrm{Au}$ as a function of studied pressure range.

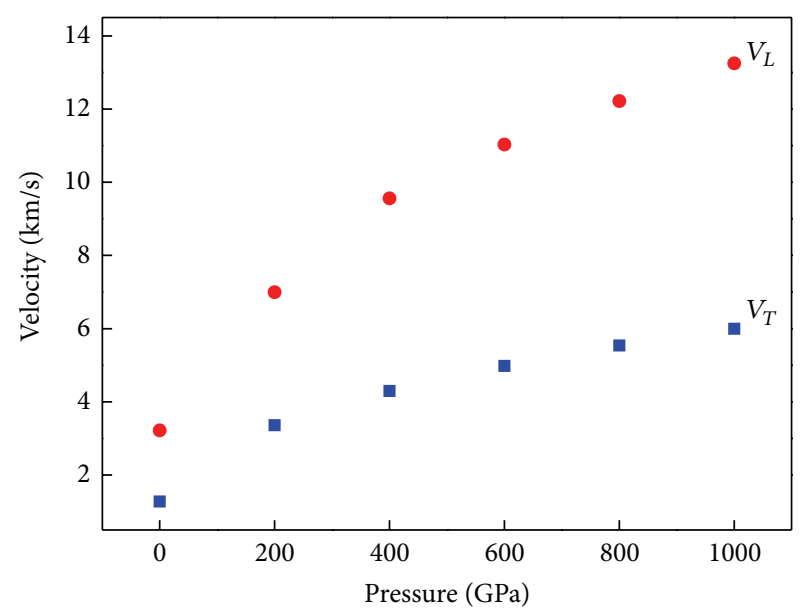

Figure 5: $V_{L}$ and $V_{T}$ behavior of Au under pressure up to $1000 \mathrm{GPa}$.

Figure 5. Under high pressure both of the sound velocities display an increment trend with the increasing of pressure; this characteristic confirms the former findings of pressure effect on both $V_{L}$ and $V_{T}$ [30]. When compared with each other $V_{L}$ has a more uniform increasing fashion than $V_{T}$.

An additional comparison is given in Tables 1 and 2 for the present typical cubic elastic constants $C_{11}, C_{12}$, and $C_{44}$ and other calculated parameters, respectively. As seen in Table 1, our obtained values for $C_{11}, C_{12}$, and $C_{44}$ are reasonable when compared with the experimental measurements. On the other hand, Table 2 summarizes the present and former experimental results (originally extrapolated from 4 Kelvin measurements) of $B, G, E, V_{L}$, and $V_{T}$ constants for $T=0$ Kelvin and $P=0 \mathrm{GPa}$. From a more physical perspective, from Tables 1 and 2, it is easy to see the existence of traditional Born mechanical (dynamical) stability conditions $(P=0 \mathrm{GPa}) ; C_{11}>0, C_{44}>0, C_{11}-C_{12}>0, C_{11}+C_{12}>0$, and $C_{12}<B<C_{11}$ [18] for Au over the entire pressure range. 
TABLE 1: Comparing previous experimental results and present calculation data for $C_{11}, C_{12}$, and $C_{44}$ constants of $\mathrm{Au}$.

\begin{tabular}{lccc}
\hline Elastic constants & $\begin{array}{l}\text { Reference } \\
{[18](\text { Exp })}\end{array}$ & $\begin{array}{l}\text { Reference } \\
{[19] \text { (Exp) }}\end{array}$ & This study \\
\hline$C_{11}(\mathrm{GPa})$ & 186.0 & 190.0 & 179.8 \\
$C_{12}(\mathrm{GPa})$ & 157.0 & 161.0 & 147.7 \\
$C_{44}(\mathrm{GPa})$ & 42.0 & 42.3 & 42.1 \\
\hline
\end{tabular}

TABLE 2: Comparing earlier experimental data of $B, E, G, V_{L}$, and $V_{T}$ values for Au with our results.

\begin{tabular}{lcc}
\hline Elastic parameters & Reference [20] (Exp) & This study \\
\hline$B(\mathrm{GPa})$ & 180.32 & 158.4 \\
$G(\mathrm{GPa})$ & 29.23 & 31.6 \\
$E(\mathrm{GPa})$ & 83.19 & 46.5 \\
$V_{L}(\mathrm{~km} / \mathrm{s})$ & 3.35 & 3.22 \\
$V_{T}(\mathrm{~km} / \mathrm{s})$ & 1.22 & 1.28 \\
\hline
\end{tabular}

Apparently our presently calculated data shows a fair consistency again for all considered parameters and their related characteristics under high pressures.

\section{Conclusion}

In summary, we surveyed the effect of pressure on several mechanical and elastic properties of Au metal with embedded atom model by geometry optimization calculations. As an outcome, the obtained findings put forth reasonable results for the high pressure behavior of Au when compared with the other experimental, molecular dynamics, and density functional results. We found that the calculated $P-V$ EOS of $\mathrm{Au}$ (Figure 1) up to $1000 \mathrm{GPa}$ is fully consistent with the former data of available experiments and theoretical results. From the high pressure point of view, with the increasing of pressure, all the elastic constants (Figures 2 and 3) and other elastic parameters (Figures 4 and 5) increased almost linearly. This finding is also compatible with the foregoing studies (Tables 1 and 2) of the considered metal. Finally, as it is obvious from all figures and tables, EAM based geometry optimization provides satisfactory results for the concerned study parameters and a convenient alternative for atomistic modeling.

\section{References}

[1] H. Kimikuza, S. Ogata, J. Li, and Y. Shibutani, "Complete set of elastic constants of $\alpha$-quartz at high pressure: a first principles study," Physical Review B, vol. 75, no. 5, Article ID 054109, 6 pages, 2007.

[2] M. Matsui, "High temperature and high pressure equation of state of gold," Journal of Physics, vol. 215, no. 1, Article ID 012197, 2010.

[3] S.-H. Shim, T. S. Duffy, and K. Takemura, "Equation of state of gold and its application to the phase boundaries near $660 \mathrm{~km}$ depth in Earth's mantle," Earth and Planetary Science Letters, vol. 203, no. 2, pp. 729-739, 2002.
[4] C. V. Pandya, P. R. Vyas, T. C. Pandya, N. Rani, and V. B. Gohel, "An improved lattice mechanical model for FCC transition metals," Physica B, vol. 307, no. 1-4, pp. 138-149, 2001.

[5] Y. Ö. Çiftci, K. Çolakoğlu, S. Kazanç, and S. Özgen, “The effect of pressure on the elastic constants of $\mathrm{Cu}, \mathrm{Ag}$ and $\mathrm{Au}$ : a molecular dynamics study," Central European Journal of Physics, vol. 4, no. 4, pp. 472-480, 2006.

[6] P. I. Dorogokupets and A. R. Oganov, "Ruby, metals, and $\mathrm{MgO}$ as alternative pressure scales: a semiempirical description of shock-wave, ultrasonic, $\mathrm{x}$-ray, and thermochemical data at high temperatures and pressures," Physical Review B, vol. 75, no. 2, Article ID 024115, 2007.

[7] J. D. Gale, "Molecular modeling theory: applications," in The Geosciences, C. R. Timothy and J. D. Kubicki, Eds., chapter 2, p. 37, Mineralogical Society of America, Washington, DC, USA, 2001.

[8] A. Budi, D. J. Henry, J. D. Gale, and I. Yarovsky, "Comparison of embedded atom method potentials for small aluminium cluster simulations," Journal of Physics Condensed Matter, vol. 21, no. 14, Article ID 144206, 2009.

[9] R. B. Wilson and D. M. Riffe, "An embedded-atom-method model for alkali-metal vibrations," Journal of Physics. Condensed Matter, vol. 24, no. 33, Article ID 335401, 2012.

[10] M. A. Tschopp, K. N. Solanki, M. I. Baskes, F. Gao, X. Sun, and M. F. Horstemeyer, "Generalized framework for interatomic potential design: application to Fe-He system," Journal of Nuclear Materials, vol. 425, no. 1-3, pp. 22-32, 2012.

[11] X. J. Zhang and C. L. Chen, "Phonon dispersion in the Fcc metals Ca, Sr and Yb," Journal of Low Temperature Physics, vol. 169, pp. 40-50, 2012.

[12] M. S. Daw and M. I. Baskes, "Semiempirical, quantum mechanical calculation of hydrogen embrittlement in metals," Physical Review Letters, vol. 50, no. 17, pp. 1285-1288, 1983.

[13] M. W. Finnis and J. E. Sinclair, "A simple empirical N-body potential for transition metals," Philosophical Magazine A, vol. 50, no. 1, pp. 45-55, 1984.

[14] E. Güler and M. Güler, "A benchmark for some bulk properties of bcc iron," The International Journal of Multiphysics, vol. 7, pp. 95-100, 2013.

[15] A. P. Sutton and J. Chen, "Long-range Finnis-Sinclair potentials," Philosophical Magazine Letters, vol. 61, pp. 139-146, 1990.

[16] J. D. Gale, "GULP: a computer program for the symmetryadapted simulation of solids," Journal of the Chemical Society, vol. 93, no. 4, pp. 629-637, 1997.

[17] J. D. Gale and A. L. Rohl, "The General Utility Lattice Program (GULP)," Molecular Simulation, vol. 29, no. 5, pp. 291-341, 2003.

[18] H. B. Huntington, The Elastic Constants of the Crystals, Academic Press, New York, NY, USA, 1958.

[19] E. A. Brandes and G. B. Brook, Smithells Metals Reference Book, Butterworth-Heinemann, Oxford, UK, 7th edition, 1992.

[20] T. M. Flynn, Cryogenic Engineering, CRC Press, 2nd edition, 2004.

[21] M. B. Taylor, G. D. Barrera, N. L. Allan, and T. H. K. Barron, "Free-energy derivatives and structure optimization within quasiharmonic lattice dynamics," Physical Review B, vol. 56, no. 22, pp. 14380-14390, 1997.

[22] C. G. Broyden, "The convergence of a class of double-rank minimization algorithms 1 . General considerations," IMA Journal of Applied Mathematics, vol. 6, no. 1, pp. 76-90, 1970.

[23] F. R. Fletcher, "New approach to variable metric algorithms," Computer Journal, vol. 13, no. 3, pp. 317-322, 1970. 
[24] D. Goldfarb, "A family of variable metric updates derived by variational means," Mathematics of Computation, vol. 24, pp. 23-26, 1970.

[25] D. F. Shanno, "Conditioning of quasi-Newton methods for function minimization," Mathematics of Computation, vol. 24, pp. 647-656, 1970.

[26] M. Güler and E. Güler, "Embedded atom method based geometry optimization aspects of body centered cubic metals," Chinese Physics Letter, vol. 30, no. 5, Article ID 056201, 2013.

[27] M. Yokoo, N. Kawai, K. G. Nakamura, K.-I. Kondo, Y. Tange, and T. Tsuchiya, "Ultrahigh-pressure scales for gold and platinum at pressures up to $550 \mathrm{GPa}$," Physical Review B, vol. 80, no. 10, Article ID 104114, 2009.

[28] T. S. Duffy, G. Shen, D. L. Heinz et al., "Lattice strains in gold and rhenium under nonhydrostatic compression to $37 \mathrm{GPa}$," Physical Review B, vol. 60, no. 22, pp. 15063-15073, 1999.

[29] C. W. Greeff and M. J. Graf, "Lattice dynamics and the high pressure equation of state of Au," Physical Review B, vol. 69, no. 5, Article ID 054107, 10 pages, 2004.

[30] H. Wang and M. Li, "The elastic stability, bifurcation and ideal strength of gold under hydrostatic stress: an ab initio calculation," Journal of Physics Condensed Matter, vol. 21, no. 45, Article ID 455401, 2009. 

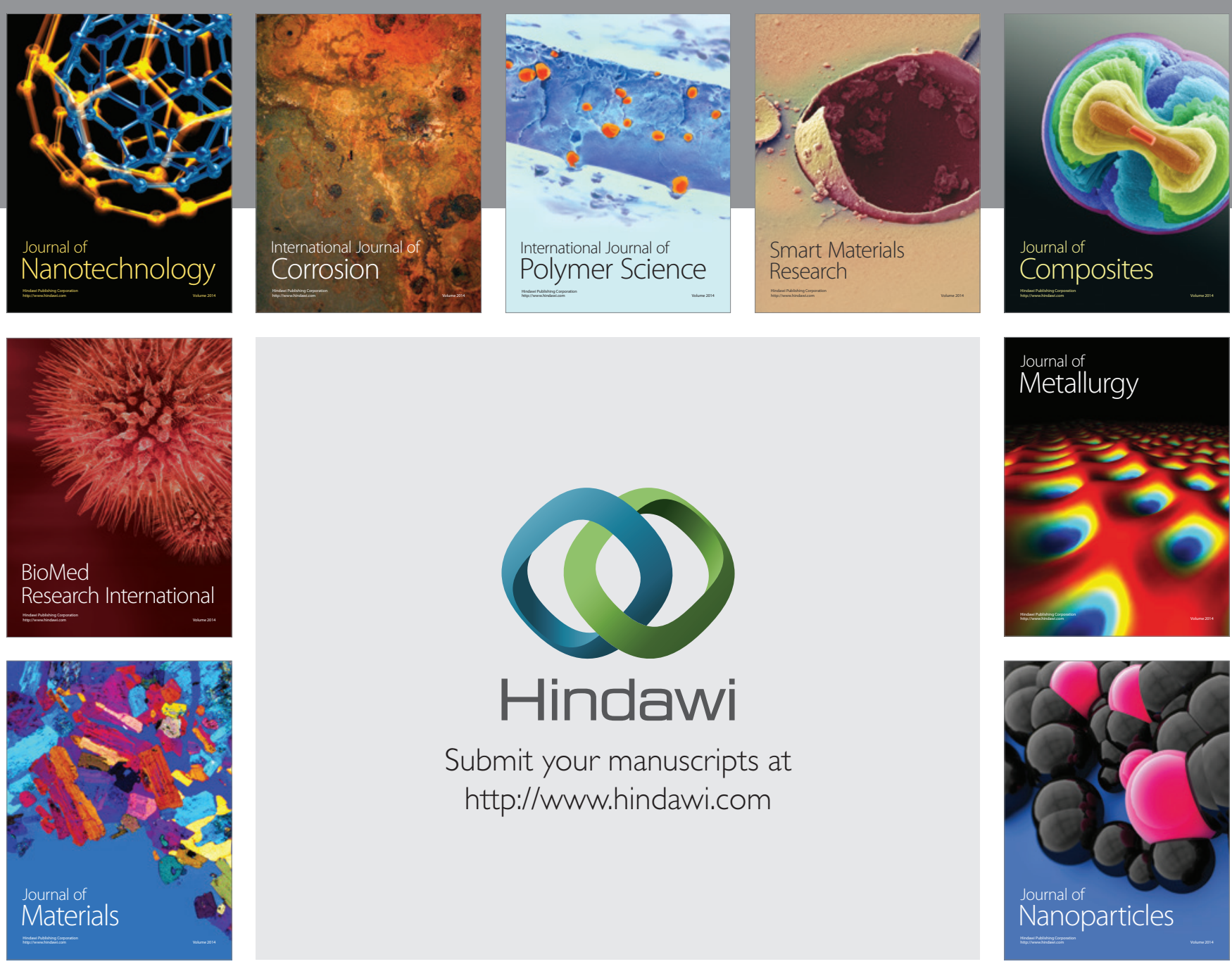

Submit your manuscripts at http://www.hindawi.com
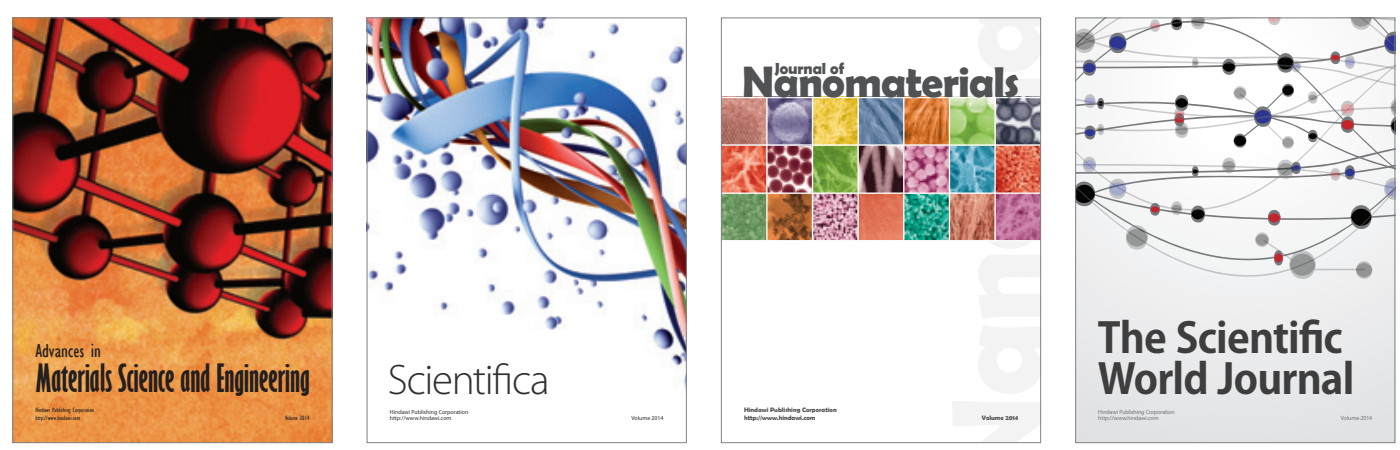

\section{The Scientific World Journal}
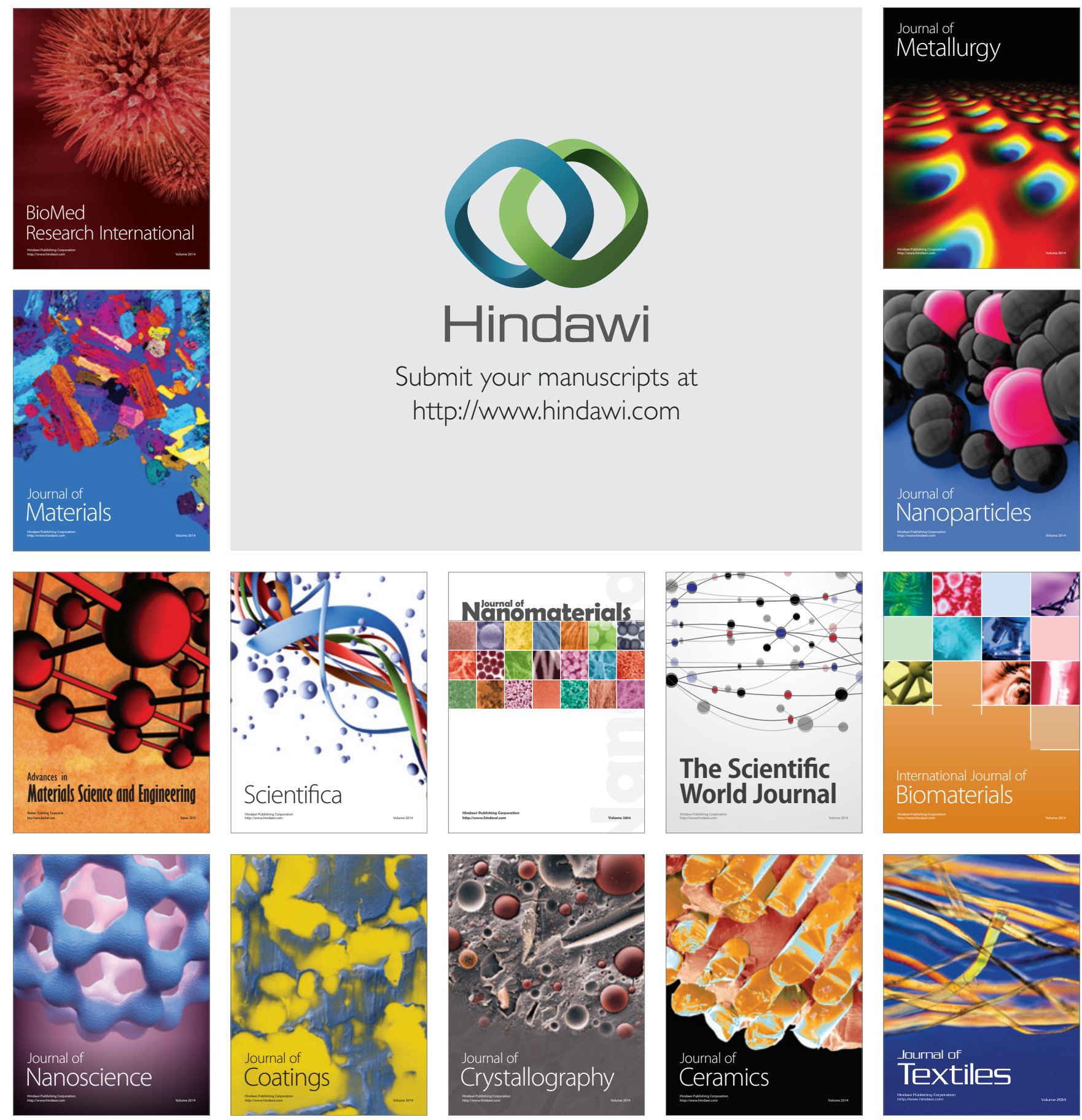